\title{
O nível de aptidão física afeta o desempenho do árbitro de futebol?
}

\author{
CDD. 20.ed. 613.7 \\ 796.022 \\ 796.33
}

\author{
Caio Max Augusto VIEIRA* \\ Eduardo Caldas COSTA** \\ Marcelo Saldanha AOKI***
}

\section{Resumo}

Os objetivos do presente estudo foram: a) descrever a demanda física imposta aos árbitros de futebol brasileiros durante partidas oficiais e b) analisar se o nivel de aptidão física interfere no desempenho da arbitragem. Os árbitros $(n=11)$ foram avaliados durante jogos oficiais $(n=21)$ do campeonato Potiguar 2009. A média de idade foi de 36,36 \pm 6,34 anos. A distância percorrida, a velocidade (média e máxima) e a frequência cardíaca (média e máxima) foram registradas durante as partidas. A análise da arbitragem foi realizada por avaliador credenciado pela Federação Norte-Rio-Grandense de Futebol (FNF), seguindo os critérios estabelecidos pela Confederação Brasileira de Futebol (CBF). A distância percorrida, a velocidade e a frequência cardiaca foram, respectivamente, 10,50 $\pm 0,35 \mathrm{~km}, 6,43 \pm 0,26 \mathrm{~km} / \mathrm{h}$ (média), 19,84 \pm 1,56 km/h (máxima), 162,77 \pm 7,44 bpm (média) e 182,22 \pm 7,72 bpm (máxima). Foi evidenciada correlação significativa entre o $\mathrm{W} 0_{2}$ máx e a distância percorrida no segundo tempo $(r=0,517)(p \leq 0,05)$. 0 W $0_{2}$ máx também apresentou correlação com a velocidade máxima de deslocamento $(r=0,506)(p \leq 0,05)$. Já o percentual de gordura apresentou correlação negativa com a velocidade máxima no segundo tempo $(r=-0,471)(p \leq 0,05)$. Foi detectada correlação positiva entre o desempenho da arbitragem e o $\mathrm{V}_{2}$ máx $(r=0,530)(p \leq 0,05)$. Com relação ao percentual de gordura, o mesmo apresentou correlação negativa com o desempenho do árbitro $(r=-0,496)(p \leq 0,05)$. Os resultados do presente estudo indicam que os árbitros de futebol são submetidos à alta sobrecarga física/fisiológica durante as partidas. Os resultados obtidos também sugerem que os parâmetros associados com a aptidão física (composição corporal e o $\mathrm{V}_{2}$ máx) podem interferir no desempenho da arbitragem.

UnItermos: Árbitros de futebol; Demanda física; Composição corporal; Consumo máximo de oxigênio.

\section{Introdução}

O futebol é um dos esportes mais praticados no mundo. Em 2003, cerca de 220 milhôes de pessoas foram consideradas membros ativos da Federation Internationale de Football Association (FIFA), sendo que 150 milhōes eram jogadores (homens e mulheres) em algum nível competitivo (CASTAGNA, ABT \& D’OTtaVIO, 2007).

Nas últimas décadas, o futebol experienciou significativo avanço do profissionalismo (GOMES \& SOUZA, 2008). Essas mudanças ocorridas nos últimos anos, principalmente em função das maiores exigências físicas, intensificaram a carga de treinamento imposta aos atletas (COHEN, ABDAlla, EjNISMAn \& AMARO, 1997). Sem dúvida, este maior nível de exigência física alterou o padrão de jogo, tornando-o mais rápido e intenso (MetaXas, Koutlianos, Kouidi \& Deligiannis, 2005).
Neste contexto do futebol moderno, é plausível assumir que a demanda física dos árbitros também tenha sofrido aumento. Para atender esse novo padrão, o desenvolvimento da aptidão física específica para o futebol se torna fundamental. E, nesse processo, merecem destaque a composição corporal, a potência aeróbia e a potência anaeróbia. Entretanto, por não fazer parte, de forma importante, da economia do futebol, a preparação dos árbitros tem sido negligenciada ao longo dos anos (CAstagna, AвT \& D'OtTavio, 2007).

Recentemente, alguns estudos avaliaram o desempenho dos árbitros em testes físicos (BARTHA, PETRIDIS, Hamar, Puhl \& Castagna, 2009; Castagna, Abt \& D’Ottavio, 2002a, 2002b, 2005; Castagna, АвT, D’Ottavio \& Weston, 2005), durante as partidas
*Departamento de Educação Física, Universidade Federal do Rio Grande do Norte;

**Programa de PósGraduação em Ciências da Saúde, Universidade Federal do Rio Grande do Norte;

***Escola de

Artes, Ciências e Humanidades, Universidade de São Paulo. 
(Castagna \& Abt, 2003; Castagna, AbT \& D’Ottavio, 2004; Castagna \& D’Ottavio, 2001; D’Ottavio \& Castagna, 2001; Mallo, Navarro, García-Aranda, Gilis \& Helsen, 2008; Tessitore, Cortis, Meeusen \& CAPRANICA, 2007) e as relações existentes entre a performance obtida nos testes físicos e a atuação durante os jogos (CAstagna, Aвt \& D'Ottavio, 2002a, 2002b; CAstagna \& D’Ottavio, 2001). No entanto, apesar do local de destaque do futebol brasileiro no cenário internacional, os estudos com árbitros brasileiros são escassos.

Castagna, AвT e D'Ottavio (2004) demonstraram que os árbitros de elite do campeonato italiano (série A) são os que percorrem maior distância durante os jogos $(-13 \mathrm{~km})$, seguidos pelos árbitros europeus (sem contabilizar os italianos) que atuam na Union of European Football Associations (UEFA) $(-11,5 \mathrm{~km})$. Alguns estudos internacionais reportaram que durante partidas oficiais os mesmos se deslocam entre 41,8 e $73,8 \%$ do tempo em baixa intensidade $(3-13 \mathrm{~km} / \mathrm{h})$, 11 a 46,3\% em média intensidade (>13-18 km/h) e 4,1 a $17,7 \%$ em alta intensidade $(>18 \mathrm{~km} / \mathrm{h}$ ) (CASTAGNA, Авт \& D'OтTAVIO, 2007). Somado a isso, a cada quatro segundos, aproximadamente, há alteração no modo de deslocamento, totalizando $\sim 1.300$ mudanças. Desse total $-45 \%$ e $-12 \%$, respectivamente, ocorrem em baixa e alta intensidade (KRUSTRUP \& BANGSBO, 2001).
No que se refere ao perfil geral do árbitro de futebol, os dados supracitados devem ser levados em consideração, visto que esses indivíduos apresentam em média 15-20 anos a mais que os jogadores. Além disto, geralmente, os mesmos atuam de forma amadora e não podem ser substituídos durante as partidas (Castagna, Aвt \& D’OtTaVio, 2007).

Portanto, diante da importância do árbitro para o jogo de futebol e da carência de trabalhos nacionais acerca da análise de desempenho desses profissionais durante partidas oficiais, o objetivo do presente estudo foi descrever o comportamento de parâmetros relacionados à demanda física (distância percorrida, velocidade e frequência cardíaca) imposta aos árbitros em jogos oficiais e verificar possíveis associações destes parâmetros com componentes da aptidão física (composição corporal e potência aeróbia). Além disso, considerando o aumento das demandas físicas do futebol, é plausível especular que o condicionamento físico dos árbitros influencie a qualidade da sua arbitragem. A fim de testar esta hipótese, o presente trabalho também investigou o nível de correlação entre a composição corporal e a potência aeróbia com o desempenho da arbitragem nas partidas, avaliado pelos critérios estabelecidos no "Manual do observador" da Confederação Brasileira de Futebol (CBF, 2007).

\section{Métodos}

\section{Amostra}

A amostra do estudo foi composta por 11 árbitros profissionais (sexo masculino; 36,36 \pm 6,34 anos; IMC $=24,54 \pm 1,80 \mathrm{~kg} / \mathrm{m}^{2}$; percentual de gordura $=16,45 \pm$ 3,90\%; نं $\mathrm{VO}_{2}$ máx $=44,98 \pm 2,67 \mathrm{ml} / \mathrm{kg} / \mathrm{min}$, e; tempo de experiência $=8,90 \pm 4,25$ anos) do quadro " $A$ " da Federação Norte-Rio-Grandense de Futebol (FNF). Todos os voluntários foram escalados para arbitrar, pelo menos, um jogo do campeonato Potiguar profissional da temporada de 2009. Os critérios de inclusão adotados foram: apresentar pelo menos três anos de experiência no quadro "A" da FNF e não apresentar nenhuma alteração osteomioarticular que limitasse parcial e/ou totalmente a realização dos testes físicos. Todos os participantes assinaram um termo de consentimento livre e esclarecido (TCLE), conforme resolução 196/96 do Conselho Nacional de Saúde. O trabalho foi aprovado pelo Comitê de Ética em Pesquisa do Hospital Universitário Onofre Lopes (HUOL) da Universidade Federal do Rio Grande do Norte (Protocolo de $n^{\circ}$ 282/09).

\section{Procedimentos}

Inicialmente, foram realizadas a avaliação da composição corporal e o teste de 2.400 metros. Isso ocorreu nos dias de treinamento comum a todos, na última semana antes do início do campeonato Potiguar de 2009. As coletas referentes à análise da demanda física nas partidas ocorreram de acordo com a escala de arbitragem determinada pela FNF, sem que isso causasse qualquer alteração no cronograma da mesma.

\section{Avaliações físicas}

Foram realizadas as medidas da massa corporal $(\mathrm{kg})$, estatura $(\mathrm{m}), \mathrm{IMC}\left(\mathrm{kg} / \mathrm{m}^{2}\right)$ e percentual de gordura. O cálculo do percentual de gordura foi efetivado por avaliador experiente através da plicometria (adipômetro Lange ${ }^{\circledR}$ científico com variação de $0,1 \mathrm{~mm}$ ), através do protocolo de JACKSON e POLLOCK (1978) de sete dobras cutâneas (subscapular, tricipital, peitoral, axilar média, supra-ilíaca, abdominal e femural média). O teste de 
2.400 metros (realizado em terreno plano) proposto por Margaria, Aghemo e Piñera Limas (1975) foi utilizado para estimar o ${ }^{\mathrm{V}} \mathrm{O}_{2}$ máx dos árbitros.

\section{Avaliação da demanda física durante as partidas}

Para avaliação da demanda física imposta aos árbitros durante as partidas foram estabelecidas as seguintes variáveis: distância percorrida $(\mathrm{km})$, velocidade média e máxima $(\mathrm{km} / \mathrm{h})$, frequência cardíaca média e máxima (bpm). Para coleta das variáveis relacionadas com a demanda física durante os jogos foi utilizado um GPS (Global Positioning System) de pulso (Garmin ${ }^{\circledR}$ modelo Forerunner 405) com cardiofrequencímetro acoplado. Os árbitros foram, previamente, instruídos sobre o manuseio do equipamento.

\section{Análise do desempenho da arbitragem}

A análise do desempenho dos árbitros foi realizada por três avaliadores oficiais credenciados pela FNF. Por haver mais de uma partida por "rodada" no campeonato, somente um avaliador foi responsável por avaliar o desempenho da arbitragem em cada partida. Como instrumento avaliativo foi usado o "Manual do observador", ferramenta padrão - instituída pela CBF - para esse propósito.

Foram contemplados três aspectos para análise de desempenho do árbitro, tendo cada um, tópicos específicos: a) aspecto técnico: 1) posicionamento com bola em jogo, 2) posicionamento com bola fora de jogo, 3) aplicação correta e coerente das regras, 4) coerência nos critérios das decisōes, 5) utilização adequada das sinalizações, 6) utilização do som do apito, 7) agilização do jogo, 8) interpretação das vantagens, 9) interpretação da indicação do impedimento e 10) integração com os árbitros assistentes e o quarto árbitro; b) aspecto disciplinar: 11) autoridade constante em campo, 12) arbitragem preventiva, 13) controle emocional, 14) aplicação correta das advertências (cartão amarelo), 15) aplicação correta das expulsões (cartão vermelho), 16) postura na aplicação dos cartôes e 17) presença nas jogadas, e; c) aspecto físico: 18) resistência física durante a partida, 19) velocidade (deslocamento rápido) e 20) aceleração.

Para avaliação do desempenho geral da arbitragem adotou-se a recomendação da CBF. Cada aspecto supracitado (técnico, disciplinar e físico) foi avaliado através de seus tópicos correspondentes. Nesse sentido, o avaliador da partida definiu um escore variando de 0-10 para cada um dos 20 tópicos supracitados (ex. aspecto técnico, tópico 1. posicionamento com bola em jogo $=7,0$ ). Após isso, todos os escores foram somados e divididos por 20, sendo definida, portanto, a nota final do árbitro na partida. No tocante à análise do aspecto físico isoladamente, os escores de resistência, velocidade e aceleração foram somados e divididos por três, obedecendo ao mesmo critério definido previamente.

\section{Análise estatística}

Para caracterização e disposição dos resultados foi utilizada a estatística descritiva (média e desvio-padrão). Todas as variáveis apresentaram distribuição normal, verificado através do método Shapiro-Wilk. Para análise de diferença (primeiro vs. segundo tempo) foi utilizado o teste t de Student pareado. Para análise de correlação das variáveis analisadas foi utilizado o coeficiente de correlação de Pearson. O pacote estatístico SPSS ${ }^{\circledR}$ versão 15.0 foi utilizado para esses fins, sendo adotado um p-valor $\leq 0,05$ como significância estatística.

\section{Resultados}

Os dados referentes à demanda física imposta aos árbitros nas partidas do campeonato Potiguar de 2009 são apresentados na TABELA 1.

Na TABELA 2 estão dispostos, separadamente, dados relacionados às exigências físicas referentes ao primeiro e ao segundo tempo de jogo. É possível observar que a velocidade média de deslocamento, assim como a frequência cardíaca média foram superiores no primeiro tempo ( $\mathrm{p} \leq 0,05)$.

Na TABELA 3 encontra-se a análise de correlação entre as variáveis relacionadas às demandas da partida e as variáveis da aptidão física (composição corporal e resistência aeróbia). Houve correlação positiva do $\mathrm{V}_{2}$ máx com a distância percorrida no segundo tempo da partida, a velocidade máxima atingida no segundo tempo de jogo e a velocidade máxima durante a partida toda $(\mathrm{p} \leq 0,05)$. O percentual de gordura apresentou correlação negativa com a velocidade máxima empregada no segundo tempo de jogo ( $\mathrm{p} \leq 0,05)$. Além disso, foi detectada correlação negativa entre o percentual de gordura e o $\dot{\mathrm{V}} \mathrm{O}_{2}$ máx $(\mathrm{p} \leq 0,01)$. 
VIEIRA, C.M.A.; COSTA, E.C. \& AOKI, M.S.

$\mathrm{Na}$ TABELA 4 são apresentados os resultados referentes à análise de correlação da composição corporal e do ${ }^{\mathrm{V}} \mathrm{O}_{2}$ máx com o desempenho da arbitragem.
É possível observar que o desempenho físico e geral dos árbitros se correlacionou, negativamente, com o percentual de gordura e, positivamente, com o $\mathrm{v}_{2}$ máx.

TABELA 1 - Demanda física imposta aos árbitros de futebol durante partidas do Campeonato Potiguar 2009.

bpm $=$ batimentos por minuto.
* = diferença estatisticamente significativa $(p-v a l o r \leq 0,05)$
$\mathrm{VO}_{2}$ máx $=$ consumo máximo de oxigênio; ${ }^{*}=p$-valor $\leq 0,05$ ${ }^{* *}=p$-valor $\leq 0,01$;
$\mathrm{VO}_{2}$ máx $=$ consumo máximo de oxigênio; ${ }^{*}=p$-valor $\leq 0,05$; ${ }^{* *}=p$-valor $\leq 0,01$;

\section{Variáveis}

Distância percorrida $(\mathrm{km})$

Velocidade média $(\mathrm{km} / \mathrm{h})$

Velocidade máxima $(\mathrm{km} / \mathrm{h})$

Frequência cardíaca média (bpm)

Frequência cardíaca máxima (bpm)
Duração da partida (min)

Média \pm Desvio-padrão

$$
\begin{gathered}
95,66 \pm 1,90 \\
10,50 \pm 0,35 \\
6,43 \pm 0,26 \\
19,84 \pm 1,56 \\
162,77 \pm 7,44 \\
182,22 \pm 7,72
\end{gathered}
$$

\begin{tabular}{|c|c|c|}
\hline & \multicolumn{2}{|c|}{ Variáveis } \\
\hline & $\%$ de Gordura ${ }^{a}$ & $\dot{\mathrm{V}} \mathrm{O}_{2}$ máx $^{\mathrm{a}}$ \\
\hline Distância $1^{\circ}$ Tempo $(\mathrm{km})$ & 0,252 & $-0,140$ \\
\hline Distância $2^{\circ}$ Tempo $(\mathrm{km})$ & $-0,429$ & $0,517^{*}$ \\
\hline Distância Total (km) & $-0,278$ & 0,376 \\
\hline Velocidade Média $1^{\circ}$ Tempo $(\mathrm{km} / \mathrm{h})$ & 0,125 & $-0,054$ \\
\hline Velocidade Média 2o Tempo $(\mathrm{km} / \mathrm{h})$ & $-0,268$ & 0,322 \\
\hline Velocidade Média Total $(\mathrm{km} / \mathrm{h})$ & $-0,086$ & 0,179 \\
\hline Velocidade Máxima $1^{\circ}$ Tempo $(\mathrm{km} / \mathrm{h})$ & $-0,148$ & 0,279 \\
\hline Velocidade Máxima $2^{\circ}$ Tempo $(\mathrm{km} / \mathrm{h})$ & $-0,471^{*}$ & $0,533^{*}$ \\
\hline Velocidade Máxima Total (km/h) & $-0,391$ & $0,506^{*}$ \\
\hline$\%$ de Gordura & - & $-0,868^{* *}$ \\
\hline
\end{tabular}

TABELA 2 - Demanda física imposta aos árbitros de futebol durante partidas do campeonato Potiguar 2009: primeiro vs. segundo tempo de jogo.

\begin{tabular}{lccc}
\hline \multicolumn{1}{c}{ Variáveis } & $\mathbf{1}^{\mathbf{0}}$ tempo & $\mathbf{2}^{\mathbf{0}}$ tempo & p-valor $^{\mathbf{a}}$ \\
\hline Duração $(\mathrm{min})$ & $46,84 \pm 1,12$ & $48,82 \pm 1,22$ & $<0,001^{*}$ \\
Distância percorrida $(\mathrm{km})$ & $5,22 \pm 0,14$ & $5,27 \pm 0,31$ & 0,577 \\
Velocidade média $(\mathrm{km} / \mathrm{h})$ & $6,57 \pm 0,40$ & $6,29 \pm 0,35$ & $0,037^{*}$ \\
Velocidade máxima $(\mathrm{km} / \mathrm{h})$ & $19,79 \pm 1,57$ & $19,89 \pm 2,11$ & 0,826 \\
Frequência cardíaca média $(\mathrm{bpm})$ & $164 \pm 7,80$ & $161,55 \pm 7,87$ & $0,040^{*}$ \\
Frequência cardíaca máxima $(\mathrm{bpm})$ & $181,85 \pm 7,17$ & $182,60 \pm 8,53$ & 0,294 \\
\hline
\end{tabular}

TABELA 3 - Correlação entre índices de demanda física nos jogos, capacidade cardiorrespiratória e composição corporal.

TABELA 4 - Correlação da capacidade cardiorrespiratória e composição corporal com desempenho de arbitragem.

\begin{tabular}{lcc}
\hline & \multicolumn{2}{c}{ Variáveis } \\
\cline { 2 - 3 } \multicolumn{1}{c}{ Desempenho da arbitragem } & \% de Gordura & 认' $\mathbf{O}_{2}$ máx \\
\hline Nota referente ao aspecto físico & $-0,758^{* *}$ & $0,748^{* *}$ \\
Nota referente ao aspecto geral & $-0,496^{*}$ & $0,530^{*}$ \\
\hline
\end{tabular}




\section{Discussão}

O presente estudo teve como objetivo descrever a demanda física imposta aos árbitros de futebol durante partidas oficiais. Além disto, outro objetivo do estudo foi investigar a influência do nível de aptidão física sobre o desempenho da arbitragem. Os principais achados foram: a) os parâmetros relacionados à demanda física da partida (distância percorrida, velocidade e frequência cardíaca) apresentaram comportamento similar em comparação ao, previamente, observado em árbitros internacionais, caracterizando o alto padrão de demanda física destes profissionais, b) o critério de avaliação da arbitragem relacionado ao aspecto físico apresentou correlação com o $\dot{\mathrm{V}} \mathrm{O}_{2}$ máx $(\mathrm{r}=0,748)$ e com o percentual de gordura $(r=-0,758)$ e c) com relação à nota geral atribuída à arbitragem, também foram observadas correlações com o $\mathrm{VO}_{2}$ máx $(\mathrm{r}=0,530)$ e com o percentual de gordura $(r=-0,496)$.

A média de idade observada foi semelhante à encontrada por DA SiLVA e RodrigueZ-AÑes (2001) em árbitros paranaenses (34,5 anos) e RoNTOYANNIS, Stalikas, SARros e Vlastaris (1998), ao avaliar árbitros gregos (36,3 anos). Esses autores apontam ainda que a idade dos árbitros, quando comparada com a dos atletas, é superior em cerca de 10 anos, e que essa diferença é menor em outros esportes. CASTAGNA, ABT e D'OtTAVIO (2007) reportam delta ainda maior nesse aspecto, diferença de 15-20 anos entre os atletas e os árbitros.

Com relação à composição corporal, o valor médio de percentual de gordura observado (16,5\%) é muito semelhante ao encontrado no trabalho de RONTOYANNIS et al. (1998) (16,7\%) e DA SiLVA e RodriguEZ-AÑES (2003) (17,3\%). Apesar do número de estudos com esse foco de investigação ser limitado, os dados disponíveis indicam que o nível de adiposidade da amostra avaliada encontra-se próximo aos índices observados na elite internacional (HELSEN \& BULTYNCK, 2004).

A respeito da demanda física imposta aos árbitros, os resultados reportados parecem corroborar os dados apresentados na literatura internacional. Tem sido relatado que os árbitros de futebol percorrem aproximadamente $10 \mathrm{~km}$ em partidas oficiais em uma intensidade média de $85-90 \%$ da frequência cardíaca máxima (Castagna, AвT e D’Ottavio, 2007).

A média da distância percorrida $(\sim 10,5 \mathrm{~km})$ pelos árbitros potiguares foi superior ao valor médio percorrido por árbitros da primeira divisão australiana e inglesa $(9,4 \mathrm{~km})$ (CATTERAll, Reilly, AtKInSON \& Coldwells, 1993; Johnson \& McNaughton, 1994), árbitros brasileiros - série A e B - $(9,15 \mathrm{~km})$
(Da Silva, Fernandes \& Fernandez, 2008) e árbitros dinamarqueses $(10 \mathrm{~km})$ (Krustrup \& BAngsbo, 2001). Entretanto, os árbitros avaliados no presente estudo apresentaram valores inferiores aos percorridos por árbitros italianos da primeira divisão do Calcio $(11,4 \mathrm{~km}$ e $13 \mathrm{~km})$ (Castagna, Авт \& D’Ottavio, 2004; D'OtTaVio \& Castagna, 2001) e europeus (não-italianos) filiados a UEFA $(11,2 \mathrm{~km})$ (CASTAGNA \& D’OtTavio, 2001). Essa distância é semelhante à percorrida pelos jogadores (meio-campistas) que mais se movimentam durante as partidas (STOLEN, Chamari, Castagna \& Wisloff, 2005).

É importante salientar que pelo fato do futebol ser uma modalidade acíclica aberta, diversos fatores devem influenciar o volume de deslocamento do árbitro na partida, tais como o padrão tático das equipes, condições climáticas, situações específicas de jogo, estado de fadiga dos jogadores, entre outros. Esses fatores poderiam explicar a grande variabilidade no padrão de deslocamento nas investigações acima citadas. Além disso, a própria condição física do árbitro influencia na quantidade de deslocamento durante os jogos (CAstagna, Aвт \& D’OTtavio, 2002a; Castagna \& D’OtTavio, 2001).

Diante da alta demanda física imposta aos árbitros, a capacidade cardiorrespiratória parece ser fundamental para o desempenho dos mesmos. Já é sabido que o $\mathrm{VO}_{2}$ máx é o melhor indicador de capacidade cardiorrespiratória (ACSM, 2000). CAstagna, ABT e D’OtTAVIo (2007) e STOLEN et al. (2005) apontam que o árbitro de futebol profissional possui em média $45-50 \mathrm{ml} / \mathrm{kg} /$ min de $\mathrm{W}_{2}$ máx. Os resultados dos árbitros potiguares encontram-se dentro desse perfil. De fato, já foi observado que o nível adequado de $\mathrm{VO}_{2}$ máx parece influenciar diretamente o desempenho físico dos árbitros durante as partidas (CASTAGna \& D’OTTAVIO, 2001). Somado a isso, dada a forte correlação encontrada entre o percentual de gordura e o $\mathrm{V}_{2}$ máx nos árbitros da FNF, a composição corporal é outro aspecto que deve ser considerado na avaliação física destes profissionais.

Castagna e D'OtTaVio (2001) verificaram que árbitros italianos com maior $\mathrm{V}_{2}$ máx percorreram maiores distâncias nos jogos, além de terem permanecido mais tempo em maior intensidade de deslocamento e menos tempo em repouso. Ainda nessa linha, Castagna, AвT e D’Ottavio (2002a) verificaram, em árbitros da comissão nacional de arbitragem da Itália, correlação positiva entre a distância percorrida em alta velocidade no segundo tempo de partidas oficiais e a distância atingida no teste de 12 minutos. Os dados 
reportados no presente estudo parecem confirmar tais achados, haja vista que houve correlação positiva entre o ${ }^{\mathrm{V}} \mathrm{O}_{2}$ máx e a distância percorrida no segundo tempo. Da mesma forma, o $\mathrm{WO}_{2}$ máx também apresentou correlação positiva com a velocidade máxima de movimentação.

A investigação referente à associação entre a potência aeróbia e o desempenho nas partidas poderia avançar, significativamente, através de padronização dos testes empregados nos estudos. No presente estudo foi utilizado o teste de $2.400 \mathrm{~m}$, pela facilidade de aplicação e interpretação dos resultados. Entretanto, no cenário internacional os trabalhos têm realizado, preferencialmente, testes intermitentes, como o "Yo-Yo Intermittent Recovery Test", por apresentar características semelhantes ao futebol (CASTAGNA, ABT \& D'OtTAVio, 2007; Krustrup, Mohr, Nybo, Jensen, Nielsen \& BANGSBO, 2006). Sem dúvida, essa é uma limitação do presente estudo. Castagna, Aвт e D'Ottavio (2005) verificaram que o teste de corrida de 12 minutos não foi capaz de detectar diferença no desempenho aeróbio de árbitros de nível alto (série $\mathrm{A}$ e $\mathrm{B}$ do campeonato italiano), médio (série C) e baixo (série D). Já o "YoYo Intermittent Recovery Test" mostrou-se bastante sensível para detectar estas diferenças.

No que diz respeito à análise dicotômica entre o primeiro e o segundo tempo de jogo, só foi verificada diferença entre a velocidade média e a frequência cardíaca média ( $\mathrm{p}$-valor $\leq 0,05$ ). Estes resultados são conflitantes aos apresentados previamente na literatura. Castagna e D’OtTaVio (2001) verificaram diferença na distância percorrida por árbitros italianos nos diferentes tempos de jogo, havendo queda de $6 \%$ na segunda etapa (5,9 vs. 5,6 km). D’OtTavio e CASTAGna (2001) avaliando árbitros da primeira divisão da Itália, observaram dados semelhantes, com diminuição de 4,1\% no segundo tempo (5,8 vs. $5,6 \mathrm{~km})$. Entretanto, DA SiLVA, Fernandes e Fernandez (2008), ao monitorar árbitros brasileiros, não encontraram diferença na distância percorrida durante a primeira e a segunda etapa (4,6 vs. $4,5 \mathrm{~km})$.

Apesar da redução na velocidade e na frequência cardíaca média dos árbitros durante o segundo tempo, a velocidade máxima e a frequência cardíaca máxima, indicadores de movimentação em alta intensidade, permaneceram semelhantes aos valores observados no primeiro tempo. Tal fato parece corroborar os achados de Castagna e D'OtTAVIO (2001) e Da Silva, Fernandes e Fernandez (2008) que não observaram diferença na distância percorrida em intensidade máxima entre o primeiro e o segundo tempo. D'Ottavio e Castagna (2001), entretanto, encontraram maior distância percorrida em velocidade máxima ( $>24 \mathrm{~km} / \mathrm{h}$ ) no segundo tempo em comparação ao primeiro tempo ( 225 vs. $202 \mathrm{~m})$.

Com relação à avaliação da arbitragem, os resultados apresentados corroboram a hipótese inicial de que a aptidão física influencia o desempenho dos árbitros. Estes dados suportam o pressuposto que a qualidade da preparação física e o consequente desenvolvimento da aptidão física desses profissionais são aspectos que podem auxiliar a atuação dos mesmos.

Sendo o futebol dependente, de forma predominante, do metabolismo aeróbio, é plausível especular que a maior potência aeróbia dos árbitros maximize sua movimentação durante a partida, permitindo o acompanhamento das jogadas de forma mais precisa. Além disso, a capacidade cardiorrespiratória adequada maximiza a recuperação entre esforços intermitentes de alta intensidade, que são característicos do futebol moderno (STOLEN et al., 2005). Isso pode facilitar o deslocamento do árbitro na partida, sem prejuízo no desempenho das ações em intensidade alta $(>18$ $\mathrm{km} / \mathrm{h}$ ) e máxima (>24 km/h) (CASTAGNA, АBT \& D’Ottavio, 2007; Krustrup \& Bangsbo, 2001).

Somado ao exposto anteriormente, o baixo percentual de gordura favorece o maior $\dot{\mathrm{W}} \mathrm{O}_{2}$ relativo, além de beneficiar açôes relacionadas ao desenvolvimento de força rápida, como sprints de curta duração (15-20 m) em velocidade máxima $(>24 \mathrm{~km} / \mathrm{h})$. Em contra partida, o excesso de peso e gordura corporal poderia afetar, negativamente, o padrão de movimentação dos árbitros e, por consequência, o julgamento das jogadas. Em lances decisivos e polêmicos, esse acompanhamento poderá determinar o resultado da partida.

É importante ressaltar que, apesar das correlaçōes observadas, uma limitação do presente estudo foi a ausência de testes mais específicos para avaliar os componentes envolvidos na aptidão para o desempenho esportivo. É plausível especular que estes componentes também apresentem relação com o desempenho dos árbitros.

Os achados do presente estudo indicam que os parâmetros associados à sobrecarga física/fisiológica da partida de futebol, analisados nos árbitros da FNF, são muito similares ao observados previamente em árbitros internacionais. Estes dados revelam que a demanda física do árbitro é muito semelhante à demanda dos jogadores que apresentam o maior nível de movimentação. No presente estudo foi observada correlação entre as notas atribuídas à arbitragem (aspecto físico e geral) e o nível de aptidão física dos árbitros. Estes dados reforçam a necessidade do treinamento físico específico direcionado a estes profissionais. 
Estudos adicionais são necessários a fim de comparar a sobrecarga física imposta aos árbitros de futebol em diferentes regiōes do Brasil, uma vez que parece existir diferenças no padrão de jogo, nas condições climáticas, no tamanho dos campos e no horário das partidas, variáveis intervenientes que podem influenciar a qualidade da arbitragem.

\section{Abstract}

Does physical fitness level affect soccer referee's performance?

The aims of the present study were to: a) report the physical demands of brazilian soccer referees during official matches and,$b$ ) assess if the level of fitness interferes in the referees' performance. The referees were examined during official games $(n=21)$ of the 2009 Rio Grande do Norte Soccer Federation Championship. The referees $(n=11)$ mean age was $36.3 \pm 6.3$ years. Match analysis parameters (distance covered, speed and heart rate) were assessed during official matches. The referee performance evaluation was conducted by an official member of Rio Grande do Norte Soccer Federation. The average match distance covered was $10.50 \pm 0.35 \mathrm{~km}$. The average speed and maximum speed were $6.43 \pm 0.26 \mathrm{~km} / \mathrm{h}$ and $19.84 \pm 1.56 \mathrm{~km} / \mathrm{h}$, respectively. Heart rate analysis revealed the intermittent nature of the referees' activities. The HRav and HRmax were $162.77 \pm 7.44$ bpm and $182.22 \pm 7.72$ bpm, respectively. There was a positive correlation $(r=0.517 ; p<0.05)$ between $\mathrm{V}_{2}$ max and distance covered on the second half. $\mathrm{V}_{2}$ max was also correlated with the maximum speed $(r=0.506 ; p<0.05)$. The fat mass was negatively correlated with maximum speed on the second half $(r=-0.471 ; p<0.05)$. Regarding referee performance, it was observed a positive correlation between ${ }^{\mathrm{V}} \mathrm{O}_{2}$ max and referee evaluation score $(\mathrm{r}=$ $0.530 ; p<0.05)$. On the other hand, fat mass was negatively correlated to referee evaluation score ( $r$ $=-0.496 ; p<0.05)$. The results present herein suggest that soccer referees were submitted to a high level of physical demands during the match. The results also indicate that soccer referees' fitness level can interfere in their performance.

UnITERMS: Soccer referees; Physical demands; Body composition; Maximum oxygen consumption.

\section{Referências}

AMERICAN COLLEGE OF SPORTS MEDICINE (ACSM). ACSM's guidelines for exercise testing and prescription. 6th ed. Baltimore: Lippincott, Williams and Wilkins, 2000.

BARTHA, C.; PETRIDIS, L.; HAMAR, P.; PUHL, S.; CASTAGNA, C. Fitness test results of Hungarian and internationallevel soccer referees and assistants. Journal of Strength and Conditioning Research, Champaign, v.23, n.1, p.121-6, 2009. CASTAGNA, C.; ABT, G. Intermatch variation of match activity in elite Italian soccer referees. Journal of Strength and Conditioning Research, Champaign, v.17, n.2, p.388-92, 2003.

CASTAGNA, C.; ABT, G.; D’OTTAVIO, S. Relation between fitness tests and match performance in elite Italian soccer referees. Journal of Strength and Conditioning Research, Champaign, v.16, n.2, p.231-5, 2002a.

. The relationship between selected blood lactate thresholds and match performance in elite soccer referees. Journal of Strength and Conditioning Research, Champaign, v.16, n.4, p.623-7, $2002 \mathrm{~b}$. Activity profile of international-level soccer referees during competitive matches. Journal of Strength and Conditioning Research, Champaign, v.18, n.3, p.486-90, 2004.

. Competitive-level differences in Yo-Yo intermittent recovery and twelve minute run test performance in soccer referees. Journal of Strength and Conditioning Research, Champaign, n.19, v.4, p.805-9, 2005.

. Physiological aspects of soccer refereeing performance and training. Sports Medicine, Auckland, v.37, n.7, p.625-46, 2007. CASTAGNA, C.; ABT, G.; D’OTTAVIO, S.; WESTON, M. Age-related effects on fitness performance in elite-level soccer referees. Journal of Strength and Conditioning Research, Champaign, v.19, n.4, p.785-90, 2005. 
CASTAGNA, C.; D'OTTAVIO, S. Effect of maximal aerobic power on match performance in elite soccer referees. Journal of Strength and Conditioning Research, Champaign, v.15, n.4, p.420-5, 2001.

CATTERALL, C.; REILlY, T.; ATKINSON, G.; COLDWELLS, A. Analysis of work rate and heart rates of association football referees. British Journal of Sports Medicine, London, v.27, n.3, p.153-6, 1993.

COHEN, M.; ABDALLA, R.J.; EJNISMAN, B.; AMARO, J.T. Lesōes ortopédicas no futebol. Revista Brasileira de Ortopedia, São Paulo, v.32, n.12, p.940-4, 1997.

CONFEDERAÇÃO BRASILEIRA DE FUTEBOL (CBF). Manual do observador. Rio de Janeiro: CBF, 2007. Disponível em: <http://www.cbf.com.br/media/1709/manual_observador.pdf>.

DA SILVA, A.I.; FERNANDES, L.C.; FERNANDEZ, R. Energy expenditure and intensity of physical activity in soccer referees during match-play. Journal of Sports Science \& Medicine, Bursa, v.7, p.327-34, 2008.

DA SILVA, A.I.; RODRIGUEZ-AÑEZ, C. Dispêndio energético do árbitro e do árbitro assistente de futebol. Revista da Educação Física/UEM, Maringá, v.12, n.2, p.113-8, 2001.

Níveis de aptidão física e perfil antropométrico dos árbitros de elite do Paraná credenciados pela Confederação Brasileira de Futebol (CBF). Revista Portuguesa de Ciências do Desporto, Porto, v.3, n.3, p.18-26, 2003.

D'OTTAVIO, S.; CASTAGNA, C. Analysis of match activities in elite soccer referees during actual match play. Journal of Strength and Conditioning Research, Champaign, v.15, n.2, p.167-71, 2001.

GOMES, A.C.; SOUZA, J. Futebol: treinamento desportivo de alto rendimento. Porto Alegre: Artmed, 2008.

HELSEN, W.; BULTYNCK, J.B. Physical and perceptual-cognitive demands of top-class refereeing in association football. Journal of Sports Sciences, London, v.22, n.2, p.179-89, 2004.

JACKSON, S.A.; POLLOCK, M.L. Generalized equations for predicting body density of men. British Journal of Nutrition, Cambridge, v.40, n.3, p.497-504, 1978.

JOHNSON, L.; McNAUGHTON, L. The physiological requirements of soccer refereeing. Australian Journal of Science and Medicine in Sport, Penneant Hills, v.26, n.3/4, p.67-72, 1994.

KRUSTRUP, P.; BANGSBO, J. Physiological demands of top-class soccer refereeing in relation to physical capacity: effect of intense intermittent exercise training. Journal of Sports Sciences, London, v.19, n.11, p.881-91, 2001.

KRUSTRUP, P.; MOHR, M.; NYBO, L.; JENSEN, J.M.; NIELSEN, J.J.; BANGSBO, J. The Yo-Yo IR2 test: physiological response, reliability, and application to elite soccer. Medicine and Science in Sports and Exercise, Madison, v.38, n.9, p.1666-73, 2006. MALLO, J.; NAVARRO, E.; GARCÍA-ARANDA, J.M.; GILIS, B.; HELSEN, W. Analysis of the kinematical demands imposed on top-class assistant referees during competitive soccer matches. Journal of Strength and Conditioning Research, Champaign, v.22, n.1, p.235-42, 2008.

MARGARIA, R.; AGHEMO, P.; PIÑERA LIMAS, F. A simple relation between performance in running and maximal aerobic power. Journal of Applied Physiology, Washington, v.38, n.2, p.351-2, 1975.

METAXAS, T.I.; KOUTLIANOS, N.A.; KOUIDI, E.J.; DELIGIANNIS, A.P. Comparative study of field and laboratory tests for the evaluation of aerobic capacity in soccer players. Journal of Strength and Conditioning Research, Champaign, v.19, n.1, p.79-84, 2005. RONTOYANNIS, G.P.; STALIKAS, A.; SARROS, G.; VLASTARIS, A. Medical, morphological and functional aspects of Greek football referees. Journal of Sports Medicine and Physical Fitness, Turin, v.38, n.3, p.208-14, 1998.

STOLEN, T.; CHAMARI, K.; CASTAGNA, C.; WISLOFF, U. Physiology of soccer: an update. Sports Medicine, Auckland, v.35, n.6, p.501-36, 2005.

TESSITORE, A.; CORTIS, C.; MEEUSEN, R.; CAPRANICA, L. Power performance of soccer referees before, during, and after official matches. Journal of Strength and Conditioning Research, Champaign, v.21, n.4, p.1183-7, 2007.

ENDEREÇO

Marcelo Saldanha Aoki

Escola de Artes, Ciências e Humanidades Universidade de São Paulo

Av. Arlindo Bettio, 1.000

03828-00o - São Paulo - SP - BRASIL

e-mail: saldanha.caf@usp.br
Recebido para publicação: 25/09/2009

Revisado em: 05/03/2010

Aceito: 15/03/2010 